\title{
Improvement of optical and electrical properties of ITO thin films by electro-annealing
}

\author{
Hasan Koseoglu a, b, *, Fulya Turkoglu a, b, Metin Kurt a, Mutlu D. Yaman a, c, \\ Fatime G. Akca ${ }^{a}$, Gulnur Aygun ${ }^{a}$, Lutfi Ozyuzer ${ }^{\mathrm{a}}$ \\ a Department of Physics, Izmir Institute of Technology, 35430, Urla, Izmir, Turkey \\ ${ }^{\mathrm{b}}$ Department of Material Science and Engineering, Izmir Institute of Technology, 35430, Urla, Izmir, Turkey \\ ${ }^{\text {c } T e k n o m a ~ T e c h n o l o g i c a l ~ M a t e r i a l s ~ L t d, ~ I Z T E K G E B, ~ I Z T E C H ~ C a m p u s, ~ U r l a, ~ I z m i r, ~ T u r k e y ~}$
}

\section{A R T I C L E I N F O}

\section{Article history:}

Received 1 December 2014

Received in revised form

26 May 2015

Accepted 16 June 2015

Available online 23 June 2015

\section{Keywords:}

ITO thin film

Electro-annealing

Large area magnetron sputtering

\begin{abstract}
A B S T R A C T
The effect of electro-annealing in vacuum and air on the optical and electrical properties of ITO thin films grown by large area DC magnetron sputtering was investigated. Moreover, the performances of the electro-annealed ITO thin films in vacuum and air were compared. Electro-annealing was performed by applying $0.75,1.00,1.25$ and 1.50 A constant ac current to the ITO thin films. It was observed that the crystallinity of the films was better for the ITO thin films electro-annealed in vacuum. The changes in sheet resistance of electro-annealed ITO thin films with applied currents were detailed. The transmittance of the films increased for both electro-annealing in vacuum and air. A correlation between band-gap and resistivity for all of the electro-annealed thin films was observed.
\end{abstract}

(C) 2015 Elsevier Ltd. All rights reserved.

\section{Introduction}

Transparent conducting oxides (TCOs) have a wide range of applications including transparent electrodes in LCDs, organic light emitting diodes, solar cells, plasma display panels (PDP), transparent heat reflecting windows as well as surface heaters for cameras, lenses, mirrors, car windows, gas sensors and ohmic contacts for surface-emitting diodes [1-3]. Indium tin oxide (ITO) thin film is one of the most commonly used materials among the TCO thin films due to its relatively low resistivity and high optical transmittance in the visible region of the electromagnetic spectrum [4].

Growth conditions such as oxygen partial pressure, substrate temperature and bias voltage are strongly important parameters for the high electrical conductivity and the transmittance in the visible spectral range of ITO films. Several deposition techniques such as thermal evaporation [5], chemical vapor deposition [6], electron beam evaporation [7], sol-gel, spray pyrolysis [8], pulsed laser deposition [9] and magnetron sputtering [10] have been used for years in order to prepare high quality ITO films. The magnetron

\footnotetext{
* Corresponding author. Department of Physics, Izmir Institute of Technology, 35430, Urla, Izmir, Turkey.

E-mail address: hasankoseoglu@iyte.edu.tr (H. Koseoglu).
}

sputtering method is the commonly preferred technique due to the reproducible deposition of the films.

Annealing of ITO thin films produced by magnetron sputtering is done to achieve the desired structure and properties of the films for practical applications [10]. The most common method for the annealing of transparent conductive oxides is thermal annealing [11]. As an alternative, recent studies have reported that the performance of deposited ITO films can be improved by electroannealing which is a process of self-heating by electric current [12-14]. Electro-annealing offers many advantages, i.e. no external heater is required during annealing and the direct Joule heat generated by applying an electric field to the film leads to a decrement in both impurity generation and heat overloading by the surrounding components [12]. Moreover, electro-annealing is suitable for thermally sensitive substrates [12], because, in the case of electro-annealing, crystallization starts and proceeds faster at lower power levels in contrast to thermal annealing due to efficient energy coupling from Joule heating. Developing a clear understanding about the characteristics of ITO thin films under electrical current flow is crucial to eliminate the life-time effects of the electronic devices. However, there is limited research concerned with the investigation of the influence of electro-annealing on the properties of the ITO thin films by passing electric currents through them in air and vacuum [12-14]. In the previous studies, Rogozin et al. [12] proposed a method which provides constant power at 
variable film resistance. They pointed out that electro-annealing in vacuum provides reduction in the thermal budget and a decrease in the kinetic exponent of crystallization in comparison with thermal annealing. Lee et al. [14] observed that optical and electrical properties of the ITO thin films on polyimide substrates can be improved by electro-annealing in air. In a recent study, Pei et al. [13] investigated the effect of electro-annealing in air on the optical and electrical properties of ITO films, and they concluded that the electric power is a decisive factor that determines the performance of the films. We report here a study of the effect of electroannealing in both air and vacuum on structural, optical and electrical properties of the ITO thin films grown by DC magnetron sputtering using borosilicate glass substrates. In contrast to the previous researches, large area substrates were used to investigate the effect of high electrical current on the ITO thin films in large scale applications.

\section{Experimental}

A large area magnetron sputtering coating system was used for the deposition of the ITO thin films [10]. The schematic representation of the large area magnetron sputtering coating system can be seen in Fig. 1. In this system, the movable sample holder is slowly moved underneath the target via a feedthrough system and the film thickness depends on the applied power and angular velocity of the feedthrough mechanism. $3 \mathrm{~mm}$ thick and $50 \mathrm{~mm} \times 60 \mathrm{~mm}$ width and length borosilicate glasses were used as substrates. They were cleaned in an ultrasonic bath in the order of acetone, alcohol and de-ionized water for $10 \mathrm{~min}$ in each and then the final cleaning was done by plasma etching for 10 min to remove organic residue. After cleaning the substrates, they were placed onto the sample holder $\left(50 \times 90 \mathrm{~cm}^{2}\right)$. To evacuate the system to below $3.33 \times 10^{-6} \mathrm{mbar}$, we used a rough pump backing a turbo-molecular pump. After evacuation of the chamber $26 \mathrm{sccm} \mathrm{Ar}$ and $1 \mathrm{sccm} \mathrm{O}_{2}$ gases were admitted using a mass flow controller, to maintain the working gas pressure at about $5.6 \times 10^{-3}$ mbar. Then, $350 \mathrm{~W}$ DC power (ADL Maris, $10 \mathrm{~kW}$ ) was applied to generate the plasma on the $50 \times 20 \mathrm{~cm}^{2}$ ITO target (purity of $99.99 \%$ and a weight per cent of 10-90 for $\mathrm{SnO}_{2}$ and $\mathrm{In}_{2} \mathrm{O}_{3}$ ). During the ITO thin film deposition, we moved the sample holder at $3.0 \mathrm{~cm} / \mathrm{min}$, and ITO thin films with the thickness of $256 \mathrm{~nm}$ were grown at $250{ }^{\circ} \mathrm{C}$ substrate temperature which was measured by a thermocouple on the sample holder.

The schematic representation of the electro-annealing process on the ITO thin films can be seen in Fig. $2.1 \mu \mathrm{m}$ thick, $5 \mathrm{~mm}$ wide and $60 \mathrm{~mm}$ length of copper plates were attached to the two opposite edges of the substrates in order to apply a constant current. After the deposition of the ITO thin films onto the borosilicate

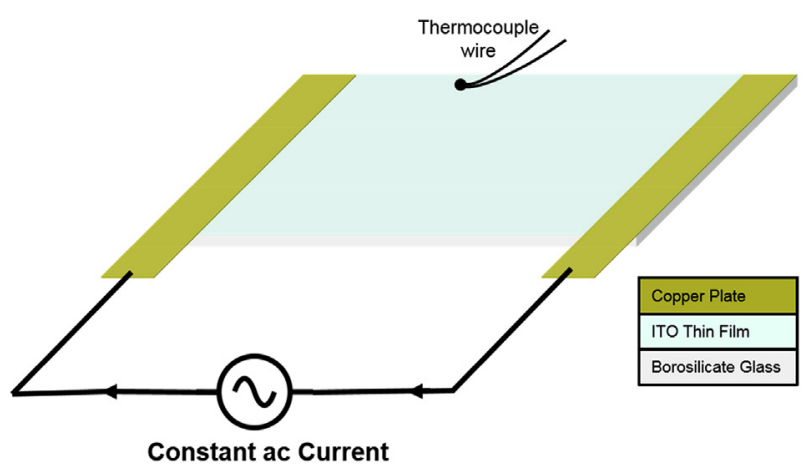

Fig. 2. Schematic representation of Electro-annealing process on ITO thin films.

glasses, electro-annealing was performed by applying $0.75,1.00$, 1.25 and 1.50 A constant ac currents to the ITO thin films in vacuum $\left(<6.6 \times 10^{-6} \mathrm{mbar}\right)$ and air for $10 \mathrm{~min}$. The ITO thin film temperature increased during electro-annealing for $10 \mathrm{~min}$ and then naturally decreased to the room temperature within between 20 and 40 min after annealing currents were switched off.

The structural properties of the ITO thin films were characterized by an X-ray diffractometer (XRD) (Philips X'Pert Pro) with $\mathrm{CuK} \alpha$ radiation $(\lambda=0.154 \mathrm{~nm})$. The optical properties of the ITO thin films were measured by a spectrophotometer (PerkinElmer Lambda 950 UV/Vis/NIR Spectrophotometer) in the 200-2600 nm wavelength range. The sheet resistances $\left(R_{S}\right)$ of ITO thin films were measured by the four point probe method using a Keithley 2425 instrument.

\section{Results and discussion}

Due to the Joule heating, the temperature of the ITO thin films increases as the electrical current passes through the films. The variation of the temperature versus time of electro-annealed thin films in air and vacuum are shown in Fig. 3. Sample temperature increased during $10 \mathrm{~min}$ and then slowly decreased to the room temperature within 20 and $40 \mathrm{~min}$. The highest temperatures for the electro-annealed ITO thin films at the end of the $10 \mathrm{~min}$ in air and vacuum were about $98,124,229,327{ }^{\circ} \mathrm{C}$ and $160,218,292$, $319^{\circ} \mathrm{C}$ when currents were $0.75,1.00,1.25,1.50 \mathrm{~A}$, respectively. Due to the heat balance between heat input and heat loss, the temperature of the films during electro-annealing in vacuum was higher as compared to electro-annealing in air for the same current input. In our set up, heat input, radiation and conductance terms of heat loss were approximately the same for electro-annealing in

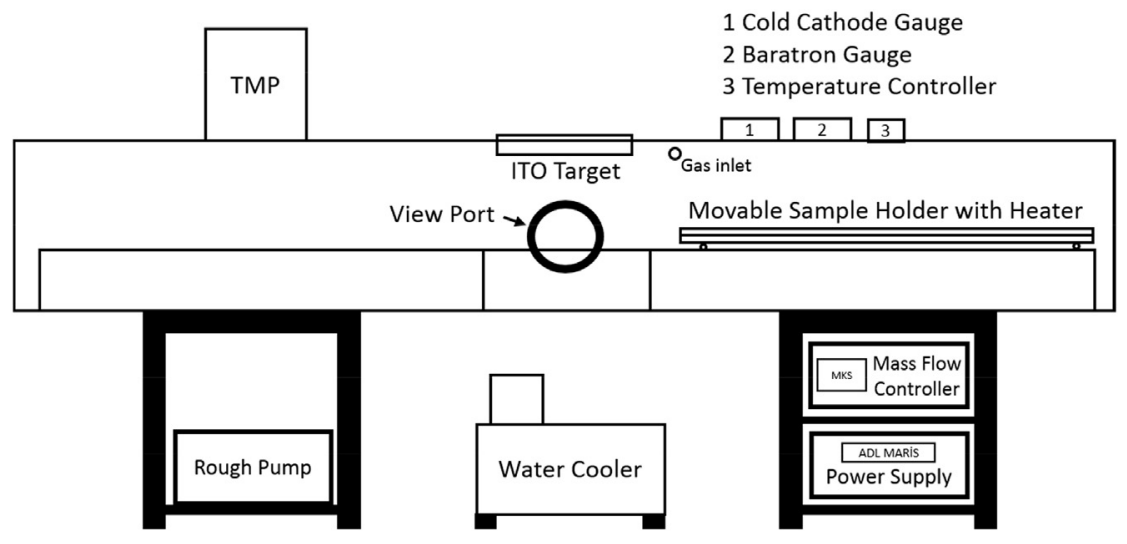

Fig. 1. Schematic representation of large area magnetron sputtering coating system. 

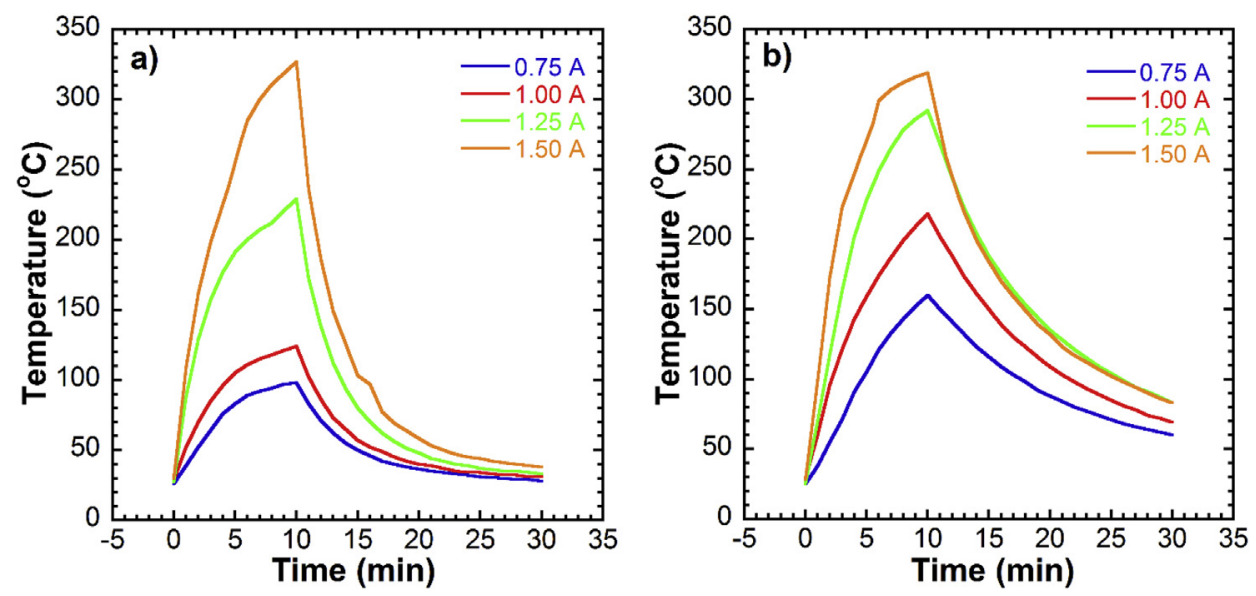

Fig. 3. The variation of temperature versus time of electro-annealed ITO thin films in a) air and b) vacuum.

vacuum and in air. However, only the convection term of heat loss was noticeably higher for electro-annealing in air, and this loss caused lower temperature of the films during electro-annealing in air.

Sheet resistances $\left(R_{S}\right)$ of the as-grown and electro-annealed ITO films in air and vacuum are shown in Fig. 4. The sheet resistances of the ITO thin films are strongly related to the amount of oxygen vacancies and microstructure [15]. The oxygen vacancies create maximum two free electrons per vacancy to the donor level through ionization, leading to an increase in conductivity. For the electro-annealing in vacuum, the $R_{S}$ of the films decreased with increasing current due to an increase of the temperature by electroannealing. The decrease in $R_{S}$ can be attributed to the improvement in the crystallinity and enhancement of oxygen vacancies which results from releasing of some oxygen atoms from the surface of the ITO thin films and the absence of free oxygen in vacuum. In comparison with the electro-annealed ITO films in vacuum, the sheet resistances of the electro-annealed ITO films in air are higher due to the reaction of free oxygen in ambient with the ITO films and less crystallization. For the electro-annealing in air, the $R_{S}$ of the films decreased up to $1.00 \mathrm{~A}$ since the lower temperature of the film

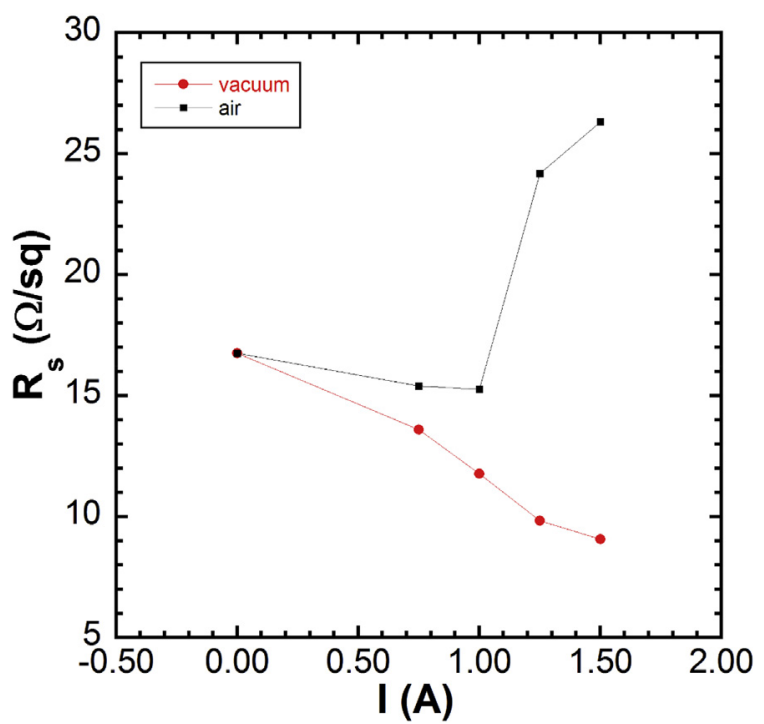

Fig. 4. Sheet resistances of electro-annealed ITO thin films in air and vacuum with applied currents. increases the interaction of oxygen atoms with In atoms and the $\mathrm{In}_{2} \mathrm{O}_{3-\mathrm{x}}$ phase is formed [13]. This formation results in the intensification of the oxygen vacancy concentration and decrease of the $R_{S}$. For the higher current values, high temperature results in interaction of the residual oxygen atoms inside the film or on the top surface with In atoms and with the $\mathrm{In}_{2} \mathrm{O}_{3-x}$ phase, after that $\mathrm{In}_{2} \mathrm{O}_{3}$ phase is formed. This formation reduces oxygen vacancy concentration and increases $R_{S}[13]$.

X-ray diffraction (XRD) was used to analyze the effect of electroannealing on the crystal structure of the ITO thin films. Fig. 5 shows XRD patterns of the as-grown (deposited at $250{ }^{\circ} \mathrm{C}$ ) and ITO thin films electro-annealed at $0.75,1.00,1.25$ and $1.50 \mathrm{~A}$ in air and vacuum. It should be noted that the broad hump between 20 and $30^{\circ}(2 \theta)$, which occurs due to background from the borosilicate glass substrate, was extracted from the XRD graphs. The XRD patterns, which consist of (211), (222), (400), (411), (431), (440) and (622) peaks, of the films indicate a cubic $\mathrm{In}_{2} \mathrm{O}_{3}$ phase. None of the spectra revealed any characteristic peaks of $\mathrm{Sn}, \mathrm{SnO}$, or $\mathrm{SnO}_{2}$ due to complete miscibility of tin atoms into the $\mathrm{In}_{2} \mathrm{O}_{3}$ lattice. It was observed that all of the ITO thin films are crystalline and have strong (222) and (400) peaks showing preferred orientations. Appearance of the strong (222) and (400) peaks indicates the coexistence of $<100\rangle$ and $<111>$ textures. For all of the electroannealed ITO thin films, it can be clearly seen that the crystallinity of the films improves by increasing the electro-annealing current. Moreover, crystalline structures become more perfect for the ITO thin films electro-annealed in vacuum. This improvement can be clearly seen in Fig. 6 which shows (222) and (400) reflections in detail for the sample electro-annealed in vacuum and in air at 1.5 A. The (222) preferred plane is a close-packed plane in the $\operatorname{In}_{2} \mathrm{O}_{3}$ body-centered cubic structure and does not accommodate vacancies very well. Unlike the (222) preferred plane, the (400) plane accommodates the oxygen vacancies on its planes. Therefore, the sheet resistances of the ITO films depend upon the orientation of the (400) plane rather than that of (222) plane. As seen in Figs. 5 and 6 and, ITO thin films electro-annealed in vacuum are more oriented along the (400) plane at the same current. This orientation also explains why the sheet resistances of the ITO thin films electroannealed in vacuum are lower than those electro-annealed in air.

Fig. 7 shows the transmittance spectra as a function of wavelength in the range of $200-2600 \mathrm{~nm}$ for the as-grown and the electro-annealed ITO thin films at different currents in air and vacuum, respectively. It clearly shows that the electro-annealing process enhances the transmittance of films in the visible region. In the visible light region, the average transmittance between 

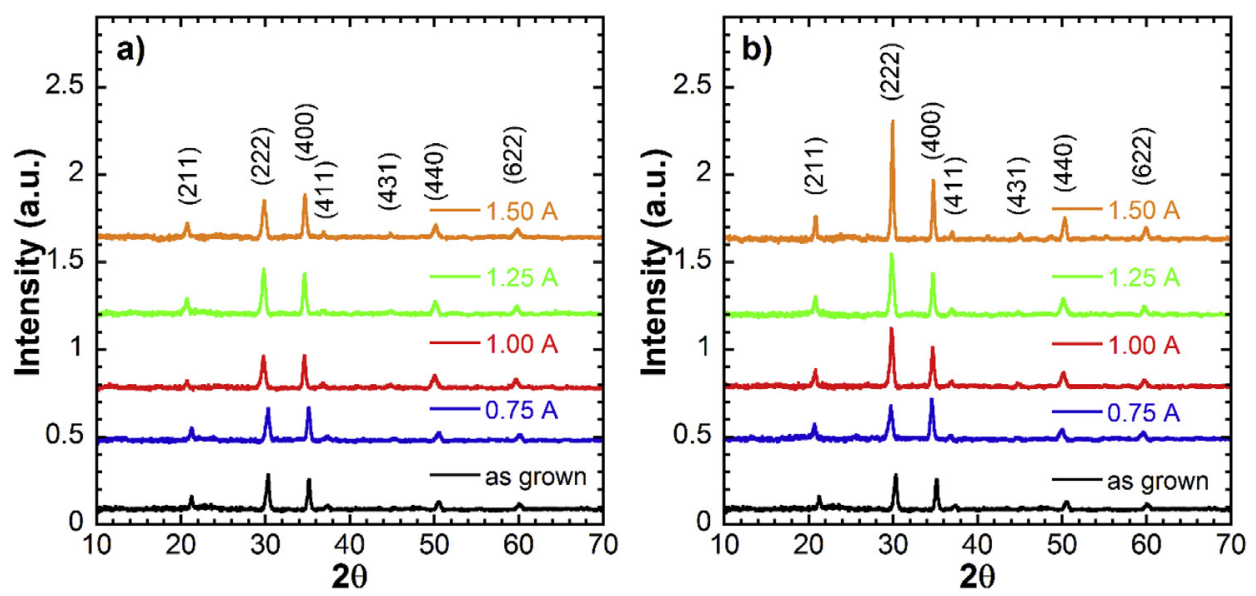

Fig. 5. XRD patterns of the as grown ITO thin films and electro-annealed ITO thin films in a) air and b) vacuum.

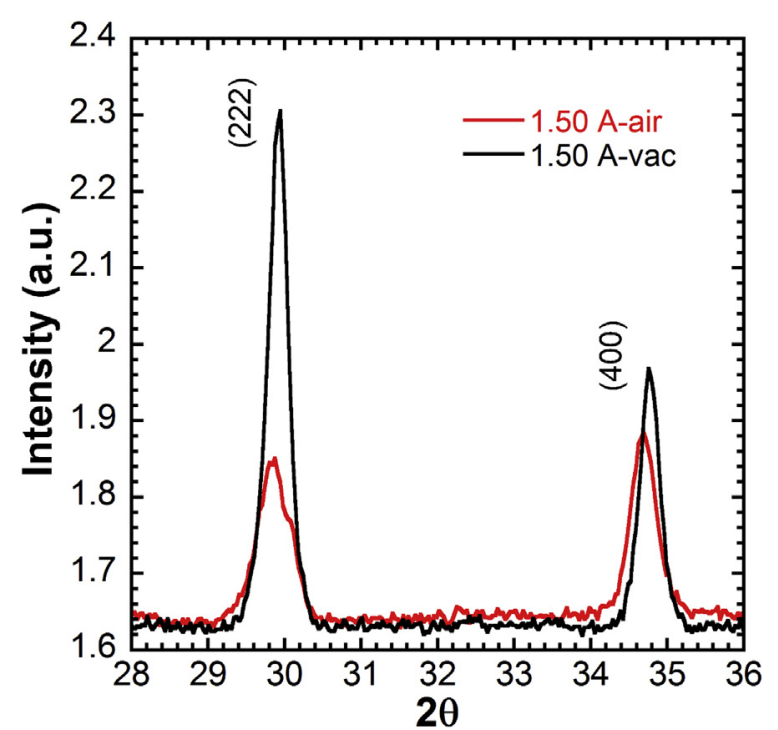

Fig. 6. Detail view of (222) and (400) reflections for the sample electro-annealed in vacuum and air at $1.5 \mathrm{~A}$.
$400 \mathrm{~nm}$ and $800 \mathrm{~nm}$ (including borosilicate glass substrate) is about $71 \%$ for the as-grown ITO thin films and it increases up to $82 \%$ both for electro-annealing in vacuum and air (Table 1). Moreover, average transmittance increases by increasing current values. Such an increase implies that the optical properties of the films improve due to the improvement of structural homogeneity of the films and the decrease of light reflection. In the near infrared region, the concentration of free carriers becomes important for the optical transmittance of the ITO films [16]. From Fig. 4, we observed that there exists a correlation between the NIR transmittance and $R_{S}$ of the films for both electro-annealing in air and vacuum. Lower resistivity values result from higher concentration of free carriers that cause higher reflection or lower transmission of the infrared region.

In the present study, the absorption coefficient, $\alpha^{2}$ versus

Table 1

Transmission, sheet resistance and energy gap results of the electro-annealed ITO thin films in air and vacuum.

\begin{tabular}{lllllll}
\hline $\mathrm{I}(\mathrm{A})$ & \multicolumn{2}{l}{ Transmission $(\%)$} & \multicolumn{2}{l}{$\mathrm{R}_{\mathrm{s}}(\Omega / \square)$} & \multicolumn{2}{l}{$\mathrm{E}_{\mathrm{g}}(\mathrm{eV})$} \\
& Air & Vacuum & Air & Vacuum & Air & Vacuum \\
\hline 0.00 & 73.497 & 73.497 & 16.761 & 16.761 & $4.280( \pm 0.027)$ & $4.280( \pm 0.027)$ \\
0.75 & 79.471 & 79.355 & 15.402 & 13.590 & $4.288( \pm 0.019)$ & $4.293( \pm 0.018)$ \\
1.00 & 79.976 & 80.486 & 15.266 & 11.778 & $4.293( \pm 0.023)$ & $4.333( \pm 0.015)$ \\
1.25 & 83.350 & 82.931 & 24.190 & 09.830 & $4.262( \pm 0.021)$ & $4.351( \pm 0.031)$ \\
1.50 & 82.744 & 82.799 & 26.319 & 09.060 & $4.235( \pm 0.022)$ & $4.373( \pm 0.013)$ \\
\hline
\end{tabular}
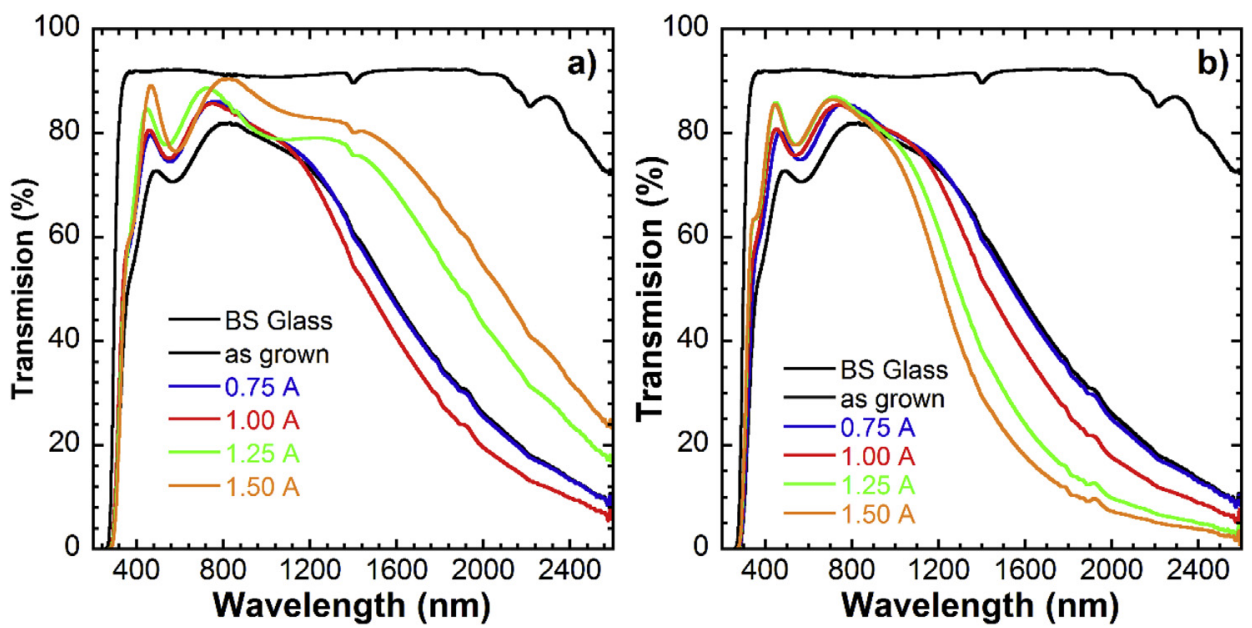

Fig. 7. Transmittance spectra as a function of wavelength in the range of $200-2600 \mathrm{~nm}$ for the as grown and electro-annealed ITO films in a) air and b) vacuum. 

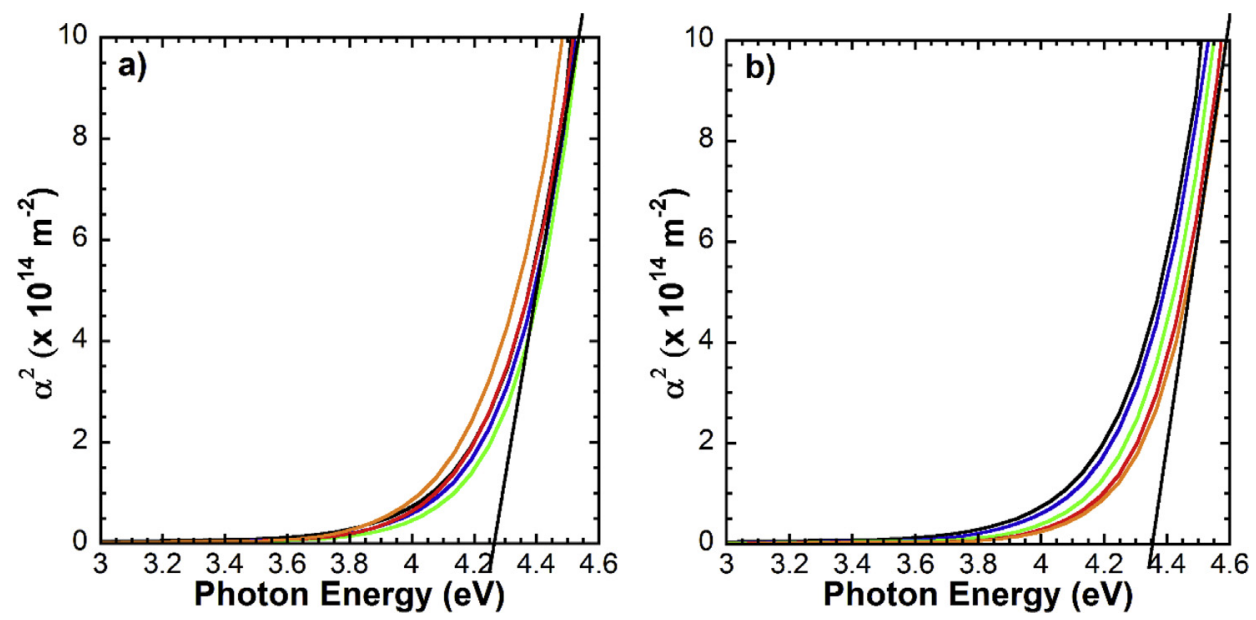

Fig. 8. $\alpha^{2}$ versus Photon Energy ( $\left.h v\right)$ curve for the as-grown and electro-annealed ITO films in a) air and b) vacuum.

photon energy ( $\mathrm{h} \nu$ ) plot was used to estimate the band-gap energy of the ITO thin films (Fig. 8). Absorption was calculated using the following equation $[17,18]$.

$T-R=e^{-\alpha t}$

where $\mathrm{T}$ and $\mathrm{R}$ denote the transmittance and reflectance, respectively, and $t$ is the thickness of the film. The reflectance of the films was neglected due to its relatively low value $[17,19]$, and then the absorption coefficient was simplified as

$\alpha^{2}=\frac{(\operatorname{InT})^{2}}{t^{2}}$

Since indium oxide has a direct transition, i.e. $\alpha h \nu=A\left(h \nu-E_{g}\right)^{1 / 2}$, the band-gaps of the films are deduced from the extrapolation of the linear plots of $\alpha^{2}$ versus $\mathrm{h} \nu$.

The direct optical band-gap values of the electro annealed ITO thin films in air and vacuum can be seen in Table 1 . The electronic gap values obtained from the fit of the curves is slightly higher for the samples annealed in vacuum compared to the samples annealed in air for the same current input. This difference may be due to the higher carrier concentrations in the samples electroannealed in vacuum. We observed that there is a correlation between the band-gap and $R_{S}$ for all of the electro-annealed thin films, i.e. while the resistivity decreases, the band-gap increases with respect to the increased annealing current. The increase in the band-gap may be due to an increase in the carrier concentrations. This band-gap widening can be explained on the basis of the Burstein-Moss shift. However, at very high carrier concentrations, there may be band-gap narrowing due to the electron-electron and electron-impurity scattering.

\section{Conclusion}

Understanding the effect of electric current on ITO thin films is essential to increase the life-time of electronic devices. In this study, we analyzed the effect of electro-annealing in air and vacuum on the structural, optical and electrical properties of the crystalline ITO thin films grown by large area DC magnetron sputtering at $250{ }^{\circ} \mathrm{C}$ substrate temperature. Both for electroannealing in air and vacuum, we observed improvement in the structural, optical and electrical properties of the ITO thin films due to the electro-annealing process. In comparison with electroannealed ITO thin films in vacuum, higher sheet resistances were obtained for the electro-annealed ITO thin films in air. Moreover, for the ITO thin films electro-annealed in vacuum, the crystallinity of the thin films was more improved compared to the thin films electro-annealed in air. The average transmittances of the films were increased up to $82 \%$ including the borosilicate glass substrate for both electro-annealing in air and vacuum. Our findings indicate that electro-annealing in vacuum is a more appropriate technique for the applications in the industry.

\section{Acknowledgments}

Dr. Basak Bengu is gratefully acknowledged for supplying borosilicate glass and the authors would like to acknowledge the facilities of Applied Quantum Research Center (AQuReC) for the current study.

\section{References}

[1] C.W. Tang, S.A. Vanslyke, Organic electroluminescent diodes, Appl. Phys. Lett 51 (1987) 913.

[2] G. Li, C.W. Chu, V. Shrotriya, J. Huang, Y. Yang, Efficient inverted polymer solar cells, Appl. Phys. Lett. 88 (2006) 253503.

[3] L. Zhao, Z. Zhou, H. Peng, R. Cui, Indium tin oxide thin films by bias magnetron rf sputtering for heterojunction solar cells application, Appl. Surf. Sci. 252 (2005) 385.

[4] H.M. Kim, K. Bae, S. Sohn, Electronic and optical properties of indium zinc oxide thin films prepared by using nanopowder target, Jpn. J. Appl. Phys. 50 (2011) 45801.

[5] D. Barreca, C. Massignan, S. Daolio, M. Fabrizio, C. Piccirillo, L. Armelao, E. Tondello, Composition and microstructure of cobalt oxide thin films obtained from a novel cobalt(II) precursor by chemical vapor deposition, Chem. Mater. 13 (2001) 588

[6] K. Ichihara, N. Inoue, M. Okubo, N. Yasuda, The origin of the inhomogeneity of electrical resistivity in magnetron-sputtered indium tin oxide thin films, Thin Solid Films 245 (1994) 152.

[7] T. Seikeb, J. Nagai, Electrochromism of 3d transition metal oxides, Sol. Energy Mater. 22 (1991) 107

[8] M. Ando, T. Kobayashi, S. Iijima, M. Harita, Optical recognition of $\mathrm{CO}$ and $\mathrm{H}_{2}$ by use of gas-sensitive $\mathrm{Au}-\mathrm{CO}_{3} \mathrm{O}_{4}$ composite films, J. Mater. Chem. 7 (1997) 1779

[9] M. Tanaka, M. Mukaia, Y. Fujimuri, M. Kondoh, Y. Tasaka, H. Baba, S. Usami, Transition metal oxide films prepared by pulsed laser deposition for atomic beam detection, Thin Solid Films 281 (1996) 453.

[10] O. Tuna, Y. Selamet, G. Aygun, L. Ozyuzer, High quality ITO thin films grown by DC and RF sputtering without oxygen, J. Phys. D: Appl. Phys. 43 (2010) 055402.

[11] L. Kerkache, A. Layadi, E. Dogheche, D. Remiens, Physical properties of RF sputtered ITO thin films and annealing effect, J. Phys. D: Appl. Phys. 39 (2006) 184.

[12] A. Rogozin, N. Shevchenko, M. Vinnichenko, M. Seidel, A. Kolitsch, W. Möller Annealing of indium tin oxide films by electric current: properties and structure evolution, Appl. Phys. Lett. 89 (2006) 061908.

[13] Y. Pei, L. Lin, W. Zheng, Y. Qu, Z. Huang, F. Lai, Effect of passing electric current on the electrical and optical properties of ITO films in air, Surf. Rev. Lett. 16 
(2009) 887 .

[14] D. Lee, S. Shim, J. Choi, K. Yoon, The effect of electro-annealing on the electrical properties of ITO film on colorless polyimide substrate, Appl. Surf. Sci. 254 (2008) 4650.

[15] W.L. Hsu, Y.H. Pai, F.S. Meng, C.W. Liu, G.R. Lin, Nanograin crystalline transformation enhanced UV transparency of annealing refined indium tin oxide film, Appl. Phys. Lett. 94 (2009) 231906.

[16] Y. Hu, X. Diao, C. Wang, W. Hao, T. Wang, Effects of heat treatment on properties of ITO films prepared by rf magnetron sputtering, Vacuum 75 (2)
(2004) 183.

[17] H.C. Lee, O.O. Park, Electron scattering mechanisms in indium-tin-oxide thin films: grain boundary and ionized impurity scattering, Vacuum 75 (2004) 275

[18] G. Sanon, R. Rup, A. Mansingh, Band-gap narrowing and band structure in degenerate tin oxide $\left(\mathrm{SnO}_{2}\right)$ films, Phys. Rev. B 44 (1991) 5672.

[19] K. Zhang, F. Zhu, C.H.A. Huan, A.T.S. Wee, Indium tin oxide films prepared by radio frequency magnetron sputtering method at a low processing temperature, Thin Solid Films 376 (2000) 255. 